\title{
WAVE PHASE IN THE OPEN-AIR LOCALIZATION OF SOUND
}

by

\section{E. Seashore}

Introduction to following articles on wove phase; open air candection; slatement of observer focts, dated rojs; oullue of kboratory problews, dated rory.

The rôle of wave phase in the localization of sound has been under investigation in our laboratory since 1908 . Interest in the subject started with a curiosity about the nature and laws of the illusion we call the phantom sound. It soon became evident that we had here an important experimental approach to the theory of sound localization. And then came the submarine. It is well known that during the great war the best means we had for locating submarines was a listening instrument in which the direction of a source of sound was located by means of the known laws of this illusion.

As chairman of the committee on acoustic problems in the National Research Council during the war period, the writer organized experimental work specifically aimed at the practical operation of this principle in the war service. Mr. H. M. Halverson was employed as research assistant and devoted his time largely to this problem for three years. The technical account of the experiments in which he took the main part is presented in the following article, to which the present article may be regarded as an introduction for the purpose of orientation. Reports of these experiments, with suggested practical application, were furnished confidentially through the National Research Council from time to time during the war.

As a bird's-eye view, or sketch of the nature and significance of this problem in "high lights," a paper read at the Boston meeting of the American Psychological Association, December, 1918, may be helpful. The facts as known ug to that date were stated as follows : 
"We undertook, as a war problem, a study of the danger of finding more than one apparently correct location of a given phantom sound. I shall here merely enumerate some of the conclusions at which we have arrived.

We have demonstrated that the most effective way to study the behavior of binaural wave phase is to dispense with the tubes or other conductors to the ear, which have previously been used in all experiments, and simply use open air conduction.

If two telephone receivers, connected in parallel from the same source of a tone, are set up within audible distance in the aural axis of the observer, then, in moving gradually from one to the other, the observer will experience the following result: For each half of a wave length there is a median plane localization which we shall call a loop; and midway between each of these is another median plane localization which we shall call a node. The difference between the two lies primarily in the fact that from the loop the phantom sound moves in the direction in which the observer moves away from tbe position at which he heard the phantom sound in the median plane; whereas, at each node it moves in the opposite direction. There are also many other differences.

Observer Seoshore

Phase Loop Node Loop Node Loop Node Loop Node Loop Node Loop Node Loop

Averace

$\begin{array}{llllllllllllll}\text { Position } & 43 & 54 & 62 & 72 & 83 & 90 & 107 & 112 & 119 & 127 & 139 & 148 & 157\end{array}$

Mean

$\begin{array}{llllllllllllll}\text { Variation } & 1.0 & 1.5 & 1.2 & 2.0 & 0.8 & 1.6 & 0.2 & 0.8 & 0.8 & 1.8 & 1.0 & 1.0 & 0.7\end{array}$

Obseruer Halurrson

Phase Loop Node Loop Node Loop Node Loop Node Loop Node Loop Node Loop

Average

Position

Mens

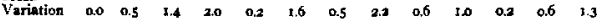

All figures are in terms of centimeters, and show distance of the hend from the beft hand source of soumd.

The phantom sound thus is experienced as moving in a series of major and minor loops." The records in Table $I$ are for a sonind of $930 \mathrm{v}$. d., the two receivers being placed two meters

1 For illustration of this see Figs. I-3 in the next following articke, by Helverson. 
apart. The wave length is here 37.4 centimeters, at $80^{\circ}$ temperature Fahrenheit. The close agreement of the two observers is to be noted.

This open air method is so superior to the previous methods, that we have followed it entirely in working out various problems. Briefly it may be said that one can demonstrate in the open air all the phenomena of wave phase as demonstrated through conductors and, in addition, many others which could not be brought under control by the earlier methods.

If the two tones beat, the observer standing at any point between the two sources will hear the phantom sound moving in an ellipse at a pace determined by the rate of the beat.

Whether the loop shall lie forward, upward, or back, is, in all cases, a matter of tendency in association, and is immaterial; i.e., as in ordinary location of sound, we cannot locate sound radially in the median plane.

If the sound in the two sources gradually rises in pitch, an endless train of loops and nodes will pass the observer. Indeed, one can make very fine pitch discriminations in terms of the movement of the phantom sound in the rise or lowering of pitch.

In many of the earlier experiments, the simple conception of a single median plane localization was due to the fact that a comparatively long wave; i.e., low pitch, was used.

The most significant thing for practical purposes is the finding that in the phantom sound the various overtones separate and each moves in its own orbit at its own peculiar rate. This timbre analysis is, indeed, very beautiful.

If you energize a telephone receiver with three of four superimposed frequencies, whether in harmonic series or not, each frequency will result in a play of its own phantom sound, and the behavior of each partial in its orbit is the same as if it were the only tone.

If we have a single rich source it instantly breaks up into the fundamental and the various overtones in remarkable purity. Thus, for example, if the telephone be put in the 60 cycle A.C. circuit, a rich tone is produced in which the first overtone is domi- 
nant and an untrained observer wift instantly locate that as the principal tone.

The significance of this is, of course, clear. While, in the submarine listening, many different conditions obtain, all devices should take into account the danger, even the certainty that a given fundamental tone will have four median plane localizations for each wave length, and that each overtone, or accessory tone, has its own orbit and its own median plane localization. The submarine listener should, theoretically, find a number of median plane localizations; not only for a given pure tone, but median localizations for each overtone, in a complex tone.

Fortunately, some of the median localizations coincide, as in any harmonic series, and it is possible to train the observer to distinguish a node from a loop, and an observer keen in tone analysis, who knows the pitch of the tone which he is to locate, may learn to disregard the intrusion of overtones.

The behavior of the phantom sound, and all its segregated harmonics, or other accessories, may be explained and interpreted in terms of the dynamics of the standing wave.

The hearing of the wave phase becomes difficult and finally impossible as one gets very close to one of the sources thereby making the intensity difference so gross that it can not be overcome by the wave phase difference.

In the open air, the wave phase effect can be obtained by the observer being placed in any direction from the two sources; that is, instead of this effect being obtainable only in the lines between the two receivers, it may be obtained anywhere within the area of audibility.

In a closed room the phenomena of reflection complicated the hearing in a nost intricate way but, of course, all in accordance with the principles of sound reflection and interference.

The size of the loops and the apparent loudness of the phantom sound vary in a very intricate but predictable way within the audible area.

The relative loudness at loop and node depends upon the ratio of the wave length to the inter-aural distance. For a long time we had difficulty from the fact that we did not realize that with 
high tones the quarter wave is so short that the crests may fall entirely within the inter-aural distance and may be lost.

In passing by small stages from one source to the other, one may hear the rise and fall of intensity in each of the components of a tone, strictly in accordance with the laws of inter ference with the standing wave phase.

We have measured empirically the flux in intensity from one receiver to another with the audioneter, and the empirical curve for observable flux in intensity corresponds with the observed movement of the phantorn sound, its apparent loudmess and distance in the various parts of its orbit.

The intensity in the two sources may vary very considerably without destroying the wave phase effect.

Both theoretically and practically we may plat a topographical map of crests and valleys representing intensity of round; and the actual behavior of the phantom sound at any place may be predicted by treating each crest as a source of sound."

So far the first public report. The program on which we were at work in the laboratory at that time may aid in throwing $\mathrm{Dr}$. Halverson's account into relief. I find the following memorandum from 1917:

The Rble of Wou-Phase Invensity in the Lacaliontion of Sound

A. Two sowrces:

1. Transition from absolute intensity to relative intensity localization.

2. Result of relative intensity due to stimulation of both ears from each side.

3. The empirical curve for flux in intensity from $L$. to $R$

4. Regult for tones composed of three elements of pitch.

5. Resull for tones of unknown pitch elements.

6. Fitch limits for localization with pure tares.

7. Eflect of absolute intensity with pure tones.

8. Efiect of absolute distance to source from the ears.

9. Effect of distance with reference to fraction of wave length.

I0. Range of effect of reflection.

If. Characteristics of passage of tone at a minimum.

12. Characteristics of passage of pure tone at a maximum.

13. Relation of open ear localization to localization in T-tubes.

14. Relation of open ear localization to beating tones with tubes.

15. Relation of open ear localization to tones in open air.

36. Limits of pitch differences in the two sources.

17. Relation of theoretical center of head to setual ear distances. 
18 The effect of varying radial axis upon the certer of the bead.

19. Gradual rige in pitch depcribed in terms of rotation of cotnd.

o. The swingtint of one source as a pendulum described in terms of rotation of sound.

21. The effect of pure tone on one side and unanalyzed rich tone on the othes,

22. The effect of fundemental on octeve; also other intervals.

23. Nodes for different kinds of noises.

34. Wave-phage in Starch's norm for perimetry of sound.

25. Relstion to confusion pointe.

26 Intra-cranial tocalization.

B. One source:

t. Verification of nodes for sownd from one gource.

2. Crucial differentiation between internal conduction and conduction around head.

3. Use one receiver and sound reflected from the wall for the other.

4 Dencribe inst of pitch in terms of rotation.

5. Effect of radial direction from center of the head.

6. Check in monaural hearing.

C. Three or more sowres:

i. Efiect of one real and one phontom gound.

x. Effect of redinl change and direction of both real and phuntom sorinds.

3. Elements of fusion.

D. Gemeral application to all norwal heoring of diretion.

E. Geisetic theory of space perception in heoring.

On the theoretical side the fundamental issue arose: does the ear have a localization mechanism for wave-phase as such, or is the wave phase localization reducible to the binaural intensity principle? Frankly we approached the subject with strong convictions in favor of the latter. For a deeper appreciation of the other alternative we are indebted to Professor G. W. Stewart, who has pursued the same subject from the point of view of physics with notable results. 